\title{
Frutos tropicais silvestres e polpas de frutas congeladas: atividade antioxidante, polifenóis e antocianinas
}

\author{
Wild fruits and pulps of frozen fruits: antioxidant activity, polyphenols and \\ anthocyanins
}

\author{
Eugenia Marta Kuskoski ${ }^{1}$ Agustín García Asuero ${ }^{2}$ \\ Maria Teresa Morales ${ }^{2}$ \\ Roseane Fett ${ }^{3}$
}

RESUMO

Os sucos de frutas tropicais conquistam cada vez mais o mercado consumidor, sendo o Brasil um dos principais produtores. Existe grande diversidade de produtos derivados de frutos e constante inserção de novos produtos no mercado de consumo, os quais, na maioria das vezes, ainda não foram devidamente pesquisados com respeito às suas propriedades $e$ atividades benéficas à saúde. Neste trabalho, objetiva-se determinar algumas propriedades de frutos tropicais silvestres in natura e polpas de frutos comercializados congelados. Para determinar a atividade antioxidante, utilizou-se o método do radical 2,2-difenil-1-picrilhidrazilo ( $\mathrm{DPPH}$ ); para determinar os polifenóis totais, o método de Folin-Ciocalteu e, para as antocianinas, o método da diferença de $p H$. As polpas de frutas analisadas foram de amora, uva, açaí, goiaba, morango, acerola, abacaxi, manga, graviola, cupuaçu e maracujá; e os frutos silvestres, jambolão e baguaçu. Representada em TEAC (atividade antioxidante equivalente a Trolox), a atividade antioxidante global das polpas oscila entre mínimos e máximos 0,5 e 53,2 $\mu \mathrm{mol} \mathrm{g}^{-1}$, enquanto que, representada em VCEAC (atividade antioxidante equivalente a vitamina $C$ ), entre mínimos e máximos de 64,8 e 1198,9mg100g ${ }^{-1}$. Para os extratos de polpa dos frutos in natura, a atividade antioxidante oscila entre 13,3 e 111,2 $\mathrm{mmol} \mathrm{g}^{-1}$ (TEAC) e entre 42,8 e 2533,1 mg $100 \mathrm{~g}^{-1}$ (VCEAC). Em ordem decrescente de capacidade antioxidante, encontra-se: acerola $>$ manga $>$ morango $>$ uva $>$ açaí $>$ goiaba $>$ amora $>$ graviola $>$ maracujá $>$ cupuaçu $>$ abacaxi. Os frutos de baguaçu apresentam maior atividade antioxidante que os de jambolão.

Palavras-chave: método $D P P H$, propriedade antioxidante, frutas tropicais.

\section{ABSTRACT}

The tropical fruit juices are gaining ever greater space in the consumer market, and Brazil is one of the main producer countries in this market. There is a great diversity of products derived from fruits and new products for consumption are launched constantly, often without the necessary research into their active properties and beneficial activities to health. The objective of this work was to determine some properties of in natura wild tropical fruit and commercialized frozen fruit pulps. Considered as a method of great applicability, 2.2difenyl-1-picrylhydrazyl radical (DPPH) was used to determine the antioxidant activity; the Folin-Ciocalteu method was used to determine the total polyphenol concentration and the $\mathrm{pH}$ difference was used for anthocyanins. The fruit pulps of greater market consumption in Southern Brazil under analysis were mulberry, grapes, açai, guava, strawberry, acerola, pineapple, mango, graviola, cupuaçu and passion fruit, and the wild fruits were jambolão and baguaçu. When represented in TEAC (Trolox equivalent antioxidant activity), the global antioxidant activity of the frozen fruit pulps oscillated between minimal and maximal TEAC values of 0.5 and $53.2 \mu \mathrm{mol} \mathrm{g}^{-1}$, or between 64.8 and

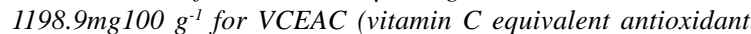
activity) values. For the in natura fruit pulp extracts, TEAC oscillated between 13.3 and $111.2 \mu \mathrm{mol} \mathrm{g}^{-1}$, and between 42.8 and $2533.1 \mathrm{mg} 100 \mathrm{~g}^{-1}$ for VCEAC. The descending order of antioxidant capacity was acerola $>$ mango $>$ strawberry $>$ grapes $>$ açaí $>$ guava $>$ mulberry $>$ graviola $>$ passion fruit $>$ cupuaçu $>$ pineapple. Among the wild fruits, baguaçu presents a greater antioxidant activity than jambolão.

Key words: $D P P H$ method, antioxidant properties, tropical fruits.

\section{INTRODUÇÃO}

O consumo de frutas tropicais aumenta ano após ano devido ao valor nutritivo e aos efeitos terapêuticos. Autilização da polpa de frutas congeladas

${ }^{1}$ Departamento de Análisis Químico y Departamento de Bioquímica, Bromatología y Toxicología, Facultad de Farmacia, Universidad de Sevilla (US) C/ García González, s/n, 41012, Sevilla, ES.

${ }^{2}$ Departamento de Análisis Químico, Facultad de Farmacia, US C/ García González s/n, 41012, Sevilla, ES.

${ }^{3}$ Departamento de Ciência e Tecnologia de Alimentos, Centro de Ciências Agrárias, Universidade Federal de Santa Catarina (UFSC). Rod. Admar Gonzaga 1346, 88034-001, Itacorubi, Florianópolis, SC, Brasil. 
está em expansão nas indústrias de produtos lácteos, de sorvetes, doces, etc., o que aumenta o interesse dos produtores e dos consumidores.

Os frutos contêm, além dos nutrientes essenciais e de micronutrientes como minerais, fibras e vitaminas, diversos compostos secundários de natureza fenólica, denominados polifenóis (HARBONE \& WILLIAMS, 2000). Inúmeros estudos realizados com compostos fenólicos, especialmente os flavonóides (antoxantinas e antocianinas), estes últimos pigmentos também presentes em vinhos tintos responsáveis pelo conhecido "paradoxo francês", demonstram a capacidade de captar radicais livres (atividade antioxidante) e seus efeitos na prevenção de enfermidades cardiovasculares e circulatórias (NESS \& POWLES, 1997; STOCLET et al., 2004), cancerígenas (WANG \& MAZZA, 2002; KATSUBE et al., 2003), no diabetes e no mal de Alzheimer (HERTOG et al., 1997; ISHIGE et al., 2001; ABDILLE et al., 2005). As mais recentes publicações relatam as propriedades de vários compostos fitoquímicos, especialmente dos compostos fenólicos presentes em frutas, atuando com eficácia nas infecções causadas por Helicobacter pylori (VATTEN et al., 2005) e na indução da apoptose (YEH \&YEN, 2005; HEO \& LEE, 2005; SÁNCHEZ-MORENO, 2002).

Devido à crescente comercialização e ao crescente consumo de frutos tropicais, tanto no mercado brasileiro como internacional, e à sua constante indicação como fonte de antioxidantes, objetiva-se neste trabalho, determinar a sua atividade antioxidante in vitro pelo método do DPPH (SÁNCHEZMORENO et al., 1998; KIM et al., 2002) e compará-la com o conteúdo de polifenóis totais e antocianinas das polpas congeladas e comercializadas no Sul do Brasil. As polpas selecionadas foram de amora (Morus nigra), uva (Vitis vinifera), açaí (Euterpe oleracea Mart.), goiaba (Psidium guajava), morango (Fragaria vesca var.), acerola (Malpighia glabra Linn.), abacaxi (Ananas comosus L.), manga (Mangifera indica L.), graviola (Anona muricato L.), cupuaçu (Theobroma grandiflorum), maracujá (Passiflora sp); e os frutos silvestres foram baguaçu (Eugenia umbelliflora Berg) e jambolão (Eugenia jambolana Lam).

\section{MATERIAL E MÉTODOS}

Utilizou-se Trolox (6-hidroxi-2,5,7,8tetrametilcromo-2-ácido carboxílico 97\%, Aldrich Chemical Co., Gillingham, Dorset, UK) como antioxidante de referência. As polpas (100\% natural) de frutas comercializadas de amora, uva, açaí, goiaba, morango, acerola, abacaxi, manga, graviola, cupuaçu e maracujá foram obtidas aleatoriamente no comércio de Florianópolis, SC Brasil, em embalagens de 100g, e conservadas sob congelamento $\left(-15 \pm 0,1^{\circ} \mathrm{C}\right)$. Os extratos das polpas de baguaçu e de jambolão foram extraídos com etanol 0,1\% de $\mathrm{HCl}$ na concentração de $10 \mathrm{~g} 25 \mathrm{~mL}^{-1}$. DPPH (2,2-difenil-1-picrilhidrazilo) e $\mathrm{FeCl}_{3}$ foram obtidos da Sigma Aldrich (Poole, Dorset, UK). As amostras de polpa foram preparadas e diluídas de acordo com a indicação para consumo (100g $250 \mathrm{~mL}^{-1}$ ) e centrifugadas. Todos os ensaios foram medidos em espectrofotômetro HP 8452A (Cheadle Heath, Stockport Cheshire, UK).

O índice de polifenóis totais foi determinado pelo método de FOLIN \& CIOCALTEU (1927), no qual a mistura dos ácidos fosfowolfrámico e fosfomolíbdico em meio básico se reduz ao oxidar os compostos fenólicos, originando óxidos azuis de wolframio $\left(\mathrm{W}_{8} \mathrm{O}_{23}\right)$ e molibdeno $\left(\mathrm{Mo}_{8} \mathrm{O}_{23}\right)$. A absorbância foi determinada a 765nm (MOYER et al., 2002). Os resultados foram expressos em mg de ácido gálico em $100 \mathrm{~g}$ de frutos.

O conteúdo de antocianinas totais foi determinado pelo método da diferença de pH (GIUSTI \& WROSLTAD, 2001), em que se dissolve em dois sistemas tampão: cloreto de potássio pH 1,0 (0,025M) e acetato de sódio $\mathrm{pH}$ 4,5 (0,4M). Foram adicionados $1,8 \mathrm{~mL}$ da correspondente dissolução tampão a $0,2 \mathrm{~mL}$ da amostra diluída (para se obter densidade óptica na faixa de 0,100-1,200, a 510nm) e efetivadas as medidas em máximos de absorção na região visível e a 700nm. A absorbância foi calculada a partir da equação:

$$
A=\left(A_{\text {max. vis }}-A_{700 \mathrm{~nm}}\right)_{\mathrm{pH} 1,0}-\left(\mathrm{A}_{\max \text { vis }}-\mathrm{A}_{700 \mathrm{~nm}}\right)_{\mathrm{pH} 4,5^{\circ}} .
$$

A concentração de pigmentos monoméricos no extrato foi calculada e representada em cianidina-3-glicosídeo. Antocianinas monoméricos (mg 100g ${ }^{-1}$ ) = $\underline{100}$

onde: $\mathrm{A}$ = absorbância; $\mathrm{PM}$ = peso molecular; $\mathrm{FD}=$ fator de diluição e $\varepsilon=$ absortividade molar.

A determinação de antocianinas foi obtida com base no volume de extrato e no peso da amostra (mg100g-1) e, calculada aplicando valores de PM: 449,2 e $\varepsilon$ : 26900, que correspondem à cianidina 3-glicosídeo. Com modificações do método descrito por KIM et al. (2002), determinou-se a absorbância do radical DPPH em 517nm. Em 3,9mL de radical DPPH a $100 \mu \mathrm{M}(3,9 \mathrm{~mL})$, dissolvido em metanol a $80 \%$, adicionou-se $0,1 \mathrm{~mL}$ da amostra (ou padrão) e foi homogeneizado cuidadosamente e guardado no escuro (30 e 60 minutos) à temperatura ambiente $\left( \pm 25^{\circ} \mathrm{C}\right)$. A absorbância foi medida a $517 \mathrm{~nm}$, antes da adição da amostra $\left(\mathrm{A}_{0}\right)$ e após 30 e 60 minutos de reação $\left(\mathrm{A}_{\mathrm{f}}\right)$. A 
concentração de DPPH no meio de reação foi calculada conforme curva de calibração obtida por regressão linear. Trolox, antioxidante sintético e hidrossolúvel similar à vitamina $\mathrm{E}$, foi preparado na concentração de 0,08-1,28mmol em metanol 80\% e determinado conforme as condições citadas anteriormente. Os resultados foram representados em TEAC, ou seja, atividade antioxidante equivalente ao Trolox $\left(\mu \mathrm{mol} \mathrm{g}{ }^{-1}\right.$ de amostra de peso da matéria fresca) e em VCEAC, atividade antioxidante equivalente à vitamina $\mathrm{C}\left(\mathrm{mg} 100 \mathrm{~g}^{-1} \mathrm{de}\right.$ peso da matéria fresca).

\section{RESULTADOS E DISCUSSÃO}

Os conteúdos totais de compostos fenólicos foram determinados como índice de polifenóis totais, representados na tabela 1 . O extrato de baguaçu contém elevado teor de polifenóis totais (897,6mg $100 \mathrm{~g}^{-1}$ ) comparados aos outros frutos em

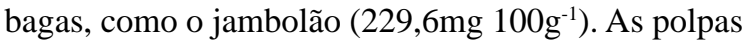
congeladas de acerola, açaí e de morango também apresentam elevados valores: 580,1mg100 $\mathrm{g}^{-1}, 136,8 \mathrm{mg}$ $100 \mathrm{~g}^{-1} \mathrm{e} 132,1 \mathrm{mg} 100 \mathrm{~g}^{-1}$, respectivamente. A quantidade de antocianinas totais nas polpas de amora, uva, morango, açaí, acerola e goiaba foram 41,8; 30,9; 23,7; 22,8; 16,0 e 2,7mg $100 \mathrm{~g}^{-1}$ peso da matéria fresca, respectivamente. Amora e uva apresentaram os maiores conteúdos de antocianinas, sendo que acerola e goiaba são as polpas que apresentaram menores conteúdos de antocianinas, enquanto que abacaxi, manga, graviola, cupuaçu e maracujá não as contêm. Nos extratos das polpas de frutos de baguaçu e jambolão, as maiores quantidades de antocianinas totais foram obtidas com etanol; valores de 596,4 e 111,2mg 100g-1, respectivamente. Extratos de baguaçu, além de apresentar elevada concentração de antocianinas, contêm os principais pigmentos, representados pela delfinidina 3-glicosídeo (17,9\%), cianidina 3-glicosídeo (1,8\%), petunidina 3-glicosídeo (15,9\%), pelargonidina 3-glicosídeo (15,9\%), peonidina 3-glicosídeo (11,7\%) e malvidina 3-glicosídeo (27,1\%) (KUSKOSKI et al., 2003).

O percentual de decréscimo da absorbância $\left(\mathrm{A}_{517}\right)$ da solução do radical DPPH foi uma resposta linear tanto em relação ao aumento da concentração Trolox ${ }^{\circledR}$ como também ao aumento da concentração de ácido ascórbico. Com base nestes resultados, os valores foram expressos em TEAC e VCEAC, respectivamente. Das amostras analisadas (polpas e extratos de polpas), os valores TEAC encontrados variam entre máximos e mínimos de 126 e $0,6 \mu$ molg $^{-1}$ para as medidas em 60 minutos (Tabela 1 ). A média dos maiores valores de TEAC foram 126; 21; 68 e 13,7 $\mu$ mol $\mathrm{g}^{-1}$ e de VCEAC foram 1623,3; 593,3; 959,1 e 174,3mg $100 \mathrm{~g}^{-1}$, correspondentes aos extratos de baguaçu, jambolão, polpa de acerola e polpa de manga, respectivamente. Os dados em VCEAC se justificam pelo fato de que as amostras são alimentos e a vitamina $\mathrm{C}$ se encontra diariamente na dieta alimentar (KIM et al., 2002), o que não significa que esteja correlacionada com o conteúdo de vitamina $\mathrm{C}$ na amostra.

Tabela 1 - Determinação de índice de polifenóis totais (IPT), antocianinas totais (AT) e atividade antioxidante (TEAC) de polpa de frutos (média $\pm \mathrm{DE}, n=3$ ).

\begin{tabular}{|c|c|c|c|c|}
\hline Amostras & IPT(mg100g ${ }^{-1}$ ) & $\mathrm{AT}^{a}\left(\mathrm{mg} 100 \mathrm{~g}^{-1}\right)$ & $\operatorname{TEAC}^{b}\left(\mu \mathrm{molg}^{-1}\right)$ & $\operatorname{TEAC}^{c}\left(\mu \mathrm{molg}^{-1}\right)$ \\
\hline Baguaçu EtOH & $897,6 \pm 5,1$ & $596,4 \pm 30,8$ & $111,2 \pm 0,1$ & $126,0 \pm 6,4$ \\
\hline Baguaçu MeOH & $704,8 \pm 27,2$ & $577,7 \pm 24,2$ & $74,1 \pm 0,4$ & $83,0 \pm 5,5$ \\
\hline Jambolão EtOH & $229,6 \pm 13,6$ & $111,2 \pm 4,1$ & $13,3 \pm 1,9$ & $20,1 \pm 1,1$ \\
\hline Jambolão MeOH & $194,7 \pm 3,5$ & $108,8 \pm 8,0$ & $15,0 \pm 3,1$ & $21,0 \pm 2,4$ \\
\hline Polpa de amora & $118,9 \pm 2,1$ & $41,8 \pm 1,8$ & $4,3 \pm 0,2$ & $5,9 \pm 0,3$ \\
\hline Polpa de uva & $117,1 \pm 0,6$ & $30,9 \pm 0,1$ & $7,0 \pm 0,3$ & $8,5 \pm 0,5$ \\
\hline Polpa de açaí & $136,8 \pm 0,4$ & $22,8 \pm 0,8$ & $6,9 \pm 0,2$ & $8,3 \pm 0,1$ \\
\hline Polpa de goiaba & $83,0 \pm 1,3$ & $2,7 \pm 0,2$ & $5,9 \pm 0,4$ & $7,4 \pm 0,1$ \\
\hline Polpa de morango & $132,1 \pm 3,8$ & $23,7 \pm 2,3$ & $9,2 \pm 0,01$ & $10,5 \pm 0,2$ \\
\hline Polpa de acerola & $580,1 \pm 4,6$ & $16,0 \pm 0,5$ & $53,2 \pm 5,3$ & $68,0 \pm 2,2$ \\
\hline Polpa de abacaxi & $21,7 \pm 4,5$ & nd & $0,5 \pm 0,01$ & $0,6 \pm 0,01$ \\
\hline Polpa de manga & $544,9 \pm 7,3$ & nd & $12,9 \pm 0,2$ & $13,7 \pm 0,4$ \\
\hline Polpa de graviola & $84,3 \pm 5,8$ & nd & $2,88 \pm 0,2$ & $4,5 \pm 0,9$ \\
\hline Polpa de cupuaçu & $20,5 \pm 3,0$ & nd & $0,73 \pm 0,2$ & $1,1 \pm 0,1$ \\
\hline Polpa de maracujá & $20,0 \pm 2,6$ & nd & $0,9 \pm 0,2$ & $1,02 \pm 0,4$ \\
\hline
\end{tabular}

${ }^{a}$ dados espectrofotométricos. ${ }^{b}$ TEAC: atividade antioxidante equivalente ao Trolox ( $\mu$ mol TE g $\mathrm{g}^{-1}$ peso da matéria fresca) em 30 minutos.

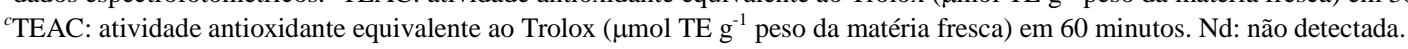


A correlação entre os valores de atividade antioxidante (TEAC), o índice de polifenóis totais e o conteúdo de antocianinas em frutos (baguaçu e jambolão) estão representados na figura 1. A média dos valores de TEAC se correlaciona de forma positiva com a média dos valores de polifenóis e antocianinas. Observa-se uma resposta tanto entre o conteúdo total de polifenóis e a atividade antioxidante dos 15 frutos analisados $\left(\mathrm{r}^{2}=0,9828, \mathrm{P}<0,01\right)$ quanto ao conteúdo total de antocianinas $(0,9381, \mathrm{P}<0,01)$. Isso indica que os compostos fenólicos são contribuintes na atividade antioxidante dos frutos analisados.

Os compostos fenólicos são os maiores responsáveis pela atividade antioxidante em frutos (HEIM et al., 2002). Embora a vitamina C seja considerada por alguns autores como o maior contribuinte na atividade antioxidante, SUN e colaboradores (2002) demonstraram que a contribuição da vitamina $C$ na determinação da atividade antioxidante de 11(onze) frutos é baixa e afirmaram que a maior contribuição para a atividade antioxidante total de frutos se deve à composição de compostos fitoquímicos. Neste trabalho, observa-se a influência dos compostos fitoquímicos na atividade antioxidante e, principalmente, a dos pigmentos antociânicos. As polpas de frutas que não os contêm, como o abacaxi, a graviola, o cupuaçu e o maracujá, apresentam valores menores de atividade antioxidante.

Entre os métodos químicos aplicados para determinar a capacidade antioxidante de um composto em capturar radicais livres, o método DPPH é um dos mais utilizados por ser considerado prático, rápido e estável (ESPIN et al., 2000). Segundo alguns autores
(ARNOUS et al., 2002; KIM et al., 2002), o tempo de medida de reação entre o radical e a amostra de 30 minutos são suficientes, embora alguns autores determinem 20 minutos (SPAGNA et al., 2002; PINELO et al., 2004). Neste trabalho, determinou-se a atividade antioxidante nos tempos de 30 e 60 minutos, com o objetivo de comprovar se existe seqüência na reação dos antioxidantes com o radical DPPH ${ }^{\circ}$. De acordo com os dados da tabela 1, os valores TEAC em 60 minutos foram mais elevados, aumentando de 10 a 50\%. A análise estatística revela diferenças significativas entre as determinações de 30 e 60 minutos, especialmente no caso das amostras de graviola, goiaba e de acerola.

Os frutos analisados neste experimento demonstram correlação direta entre o conteúdo total de compostos fenólicos e a atividade antioxidante. Contudo, para se estabelecer uma relação direta de cada grupo ou composto fenólico, será necessário um estudo específico com os compostos isolados, assim como para se determinar as quantidades diárias necessárias e ideais de antioxidantes na alimentação equilibrada, levando em consideração diversas variáveis e condições de vida de cada indivíduo. Entretanto, observa-se neste estudo que, mesmo congelados, os frutos e polpas mantiveram suas propriedades, podendo ser excelentes fontes de compostos fenólicos com capacidades antioxidantes.

\section{CONCLUSÃO}

As polpas de frutos tropicais comercializadas na forma congelada no sul do Brasil contêm elevados teores de polifenóis totais e

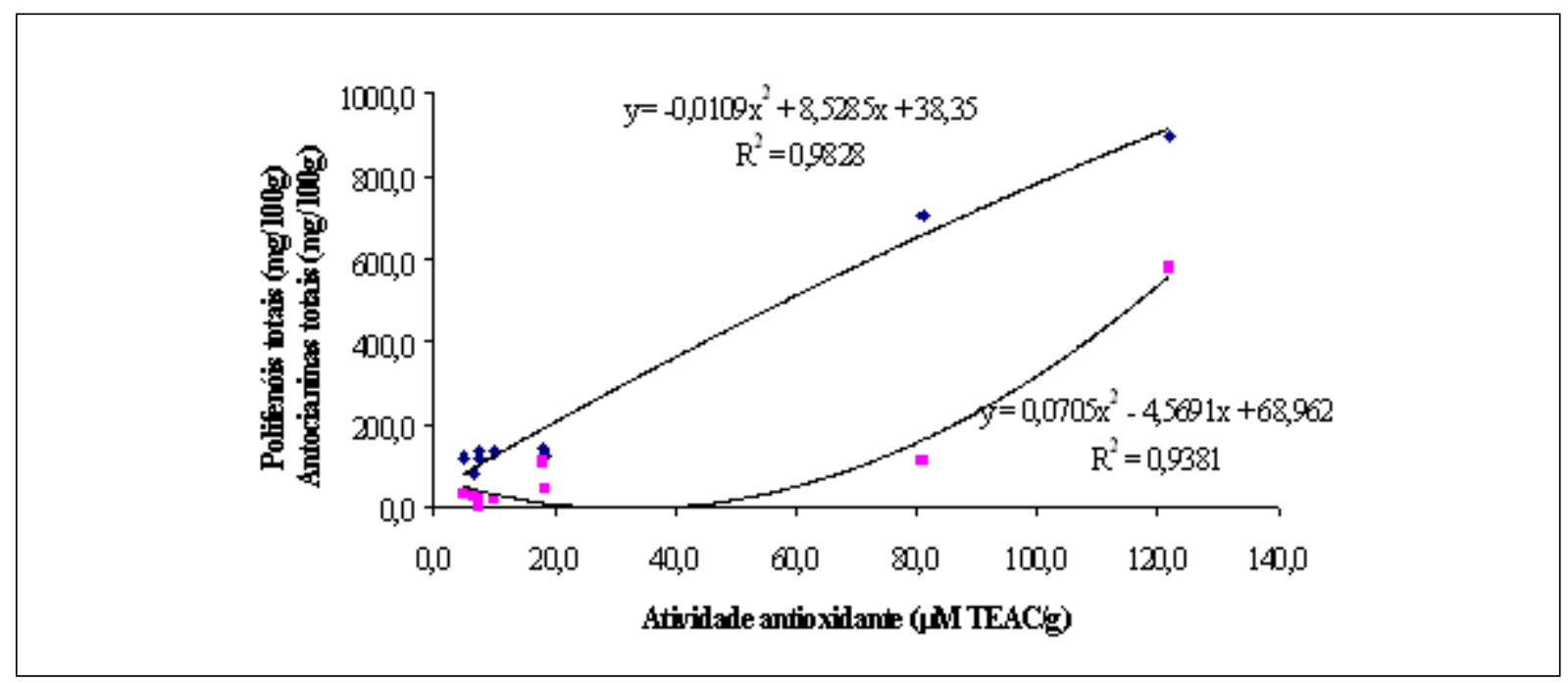

Figura 1 - Correlação entre polifenóis totais $\left(r^{2}=0,98\right)$ e antocianinas totais $\left(r^{2}=0,93\right)$ como os valores de atividade antioxidante em TEAC $\left(\mu \mathrm{molg}^{-1}\right)$.

Ciência Rural, v.36, n.4, jul-ago, 2006. 
apreciáveis propriedades antioxidantes, obtendo de maior destaque entre as polpas congeladas analisadas as de acerola e manga. Entre os frutos in natura, o baguaçu se destaca como potente antioxidante, com considerável teor de antocianinas. Há correlação direta entre os valores de polifenóis totais, antocianinas e os valores TEAC e VCEAC. Os valores de atividade antioxidante representados em TEAC demonstram ser dependentes do tempo de medida, apresentando aumentos de 10 a $50 \%$.

\section{REFERÊNCIAS}

ABDILLE, M.H. et al. Antioxidant activity of the extracts from Dillenia indica fruits. Food Chem, v.90, p.891-896, 2005.

ARNOUS, A. et al. Correlation of pigment and flavanol content with antioxidant properties in selected aged regional wines form Greece. J Food Comp Anal, v.15, p.655-665, 2002.

BRAND-WILLIAMS, et al. Use of free radical method to evaluate antioxidant activity. Lebensm Wiss Technol, v.22, p.25-30, 1995.

ESPIN, J.C. et al. Anthocyanin-based natural colorants: A new source of antiradical activity for foodstuff. J Agric Food Chem, v.48, p.1588-1592, 2000.

FOLIN, C.; CIOCALTEU, V. Tyrosine and tryptophan determination in proteins. J Biol Chem, v.73, p.627-650, 1927.

GIUSTI, M.M.; WROLSTAD, R.E. Anthocyanins: characterization and measurement with uv-visible spectroscopy. In: WROLSTAD, R.E. Current protocols in food analytical chemistry. New York: John Wiley \& Sons, 2001. Unit. F1.2.1-13.

HARBORNE, J.B.; WILLIAMS, C.A. Advances in flavonoid research since 1992. Phytochemistry, v.52, p.481-504, 2000.

HEIM, K.E. et al. Flavonoid antioxidants: chemistry, metabolism and structure-activity relationships. J Nutr Biochem, v.13, p.572-584, 2002.

HEO, H.J.; LEE, C.Y. Strawberry and its anthocyanins reduce oxidative stress-induce apoptosis in PC12 cells. J Agric Food Chem, v.53, p.1984-1989, 2005.

HERTOG, M.G.L. et al. Antioxidant flavonols and ischaemic heart disease in a Welsh population of men. The Caerphilly study. Am J Clin Nutr, v.65, p.1489-1494, 1997.

ISHIGE, K. et al. Flavonoids protect neuronal cells from oxidative stress by three distinct mechanisms. Free Rad Biol Med v.30, p.433-446, 2001.
KATSUBE, N. et al. Induction of apoptosis in cancer cells by bilberry (Vaccinium mirtillus) and the anthocyanins. J Agric Food Chem, v.51, p.68-75, 2003.

KIM, D-O. et al. Vitamin C equivalent antioxidant capacity (VCEAC) of phenolics phytochemicals. J Agric Food Chem, v.50, p.3713-3717, 2002.

KUSKOSKI, E.M. et al. Characterization of anthocyanins from the fruits of baguaçu (Eugenia umbelliflora Berg). J Agric Food Chem, v.51, p.5450-5454, 2003.

MOYER, R.A. et al. Anthocyanins, phenolics, and Antioxidants capacity in diverse small fruits: Vaccinium, Rubus, and Ribes. J Agric Food Chem, v.50, p.519-525, 2002.

NESS, A.R.; POWLES, J.W. Fruit and vegetables, and cardiovascular disease: a review. Int J Epidemiol, v.26, n.1, p.1-13, 1997.

PINELO, M. et al. Interaction among phenolics in food fortification: negative synergism on antioxidant capacity. J Agric Food Chem, v.52, p.1177-1180, 2004

SÁNCHEZ-MORENO, C. Compuestos polifenólicos: efectos fisiológicos. Actividad antioxidante. Alimentaria, p.29-40, 2002.

SÁNCHEZ-MORENO, C. et al. A procedure to measure the antiradical efficiency of polyphenols. J Sci Food Agric, v.76, p.270-276, 1998.

SPAGNA, G. et al. Chemical analysis and photoproctetive effect of an extract of wine from Jacquez grapes. J Sci Food Agric, v.82, p.1867-1874, 2002.

STOCLET, J.C. et al. Vascular protection by dietary polyphenols. Eur J Pharm, v.500, p.299-313, 2004.

SUN, J. et al. Antioxidant and antiproliferative activities of common fruits. J Agric Food Chem, v.50, p.7449-7454, 2002.

VATTEM, D.A. et al. Cranberry synergies for dietary management of Helicobacter pylori infections. Process Biochem, v.40, p.1583-1592, 2005

WANG, J.; MAZZA, G. Effects of anthocyanins and other phenolic compounds on the production of tumor necrosis factor alpha in LPS/IFN-gamma-activated RAW 264.7 macrophages. J Agric Food Chem, v.50, p.4183-4189, 2002.

YEH, C.T.; YEN G.C. Induction of apoptosis by the anthocyanidins through regulation of $\mathrm{Bcl}-2$ gene and activation of c-jun n-terminal kinase cascade in hepatoma cells. J Agric Food Chem, v.53, p.1740-1749, 2005. 